\title{
A uncanny potential of plants for metal nanoparticles synthesis
}

\begin{abstract}
Nowadays there is an imperative demand to build up reliable, sustainable, and ecofriendly protocols for manufacturing a large collection of metal and metal oxide nanoparticles. The green synthesis of those nanomaterials has gained considerable attention as a promising tool to obstruct the harmful effects of nanoparticles resulting from conservative synthesis approach. Plant extracts in particular have been extensively utilized for the preparation of metal nanoparticles with the aid of essential phytochemicals in plant extracts. This short review describes the importance of plants as green sources for synthesis of metal nanoparticles.
\end{abstract}

Volume 7 Issue 3 - 2018

\author{
Jagpreet Singh,' Harman Kaur, ${ }^{2}$ Mohit Rawat' \\ 'Department of Nanotechnology, Sri Guru Granth Sahib World \\ University, India \\ ${ }^{2}$ Department of Biotechnology, Lyallpur Khalsa College, India
}

\author{
Correspondence: Mohit Rawat, Department of \\ Nanotechnology, Sri Guru Granth Sahib World University, \\ Fatehgarh Sahib-140406, Punjab, India, \\ Email mohitnano.nit@gmail.com
}

Received: June 18, 2018 | Published: June 29, 2018

\section{Introduction}

In this era, metal nanoparticles have captivated researchers because of their impending applications in numerous fields such as biomedical, catalysis, electronics etc. ${ }^{1-4}$ The properties of nanoparticles can be tuned by their size, synthesis process, reaction parameters, which make them the special candidate for every field. Generally, these nanoparticles are synthesized by two methods top down and bottom up. The top-down suggests the nanoparticles preparation by lithographic techniques, ball milling, etching, sputtering, etc. The most effective approach for the synthesis of nanoparticles is the bottom-up methods, in which nanoparticles are grown from simpler molecules and size or shape of nanoparticles can be controlled or modulated by changing the concentration of chemicals and reaction condition (temperature, $\mathrm{pH}$ etc.). ${ }^{5}$

\section{The prerequisite}

Although there are innumerable methods for nanoparticles preparation, but theses conventional methods associated with many shortcomings like the use of noxious and expensive chemicals and complex systems. ${ }^{6}$ These limitations obstruct the demand of conventional methods for nanoparticles synthesis. Thus, there is strong need of reliable, eco-friendly and cost-effective mean for the synthesis of metal nanoparticles.

\section{Potential of plants}

To overcome the problems associated with conventional techniques green approaches comes to play a vital role. Use of various plants for synthesis of nanoparticles referred to as Green Nanotechnology. ${ }^{7}$ Generally, the phytochemicals present in plant extracts are responsible for the reduction of metal salts into respective metal nanoparticles. ${ }^{8}$ Also, the phytochemicals in plant extract stabilize the nanoparticles itself without the need of any other stabilizer. Are act as a stabilizer as well. So, plant extracts paly a dual role in nanoparticles synthesis. Till date, there are various reports available in which many plant extracts were utilized for nanoparticles synthesis..$^{9-13}$ Likewise, Savithramma et al. ${ }^{14}$ studied the antimicrobial activity of silver nanoparticles (Ag NPs) synthesized using medicinal plants were studied. Their results indicated that the Ag NPs exhibited maximum inhibition on the growth of Pseudomonas and Rhizophus species. Vijayakumar et al., ${ }^{15}$ have reported the biosynthesis, characterization and antibacterial effect of plant mediated silver nanoparticles using Artemisia nilagirica. The average size of Ag NPs was 70-90 nm and the results showed that the microbial susceptibility to Ag NPs was different for each organism studied. Ramgobal et al., ${ }^{16}$ synthesized of Ag NPs using soap nuts was performed by UV-Vis, SEM, TEM and EDX. They have reported that the Ag NPs showed more inhibitory activity on pathogenic gram positive than gram negative bacteria. Gunalan et al., ${ }^{17}$ reported the $\mathrm{ZnO}$ NPs were green synthesized by using aloe leaf broth extract. Their results indicated enhanced biocidal activity against various pathogens when compared to chemically synthesized ZnO NPs. They have also reported that the effectiveness of nanoparticles increased with increased particle dose, treatment time and synthesis method. Thus, the concept of green synthesis was introduced to nanoparticles synthesis strategy to mitigate the use of noxious and expensive chemicals.

\section{Conclusion}

In recapitulate, the synthesis of metal nanoparticles using plant extract is a reliable, cost-effective, eco-friendly and simple method. Also, the biological properties like antimicrobial, anticancer properties of nanoparticles can be enhanced through the utilization of medicinal plants. Because, plants itself have many medicinal properties. In future, we can use this uncanny potential of plants for the synthesis of different types of nanomaterials and their implications towards the nanomedicine research.

\section{Acknowledgments}

This work was financially supported by Shromani Gurdwara Prabhandak Committee (SGPC), Amritsar. Authors are thankful ViceChancellor, SGGSW University in India for providing necessary laboratory facilities. Jagpreet Singh and Harman Kaur have equally contributed to this work.

\section{Conflict of interests}

All the authors have declared that there is no conflict of interests regarding the publication of this article. 


\section{References}

1. Huang X, El-Sayed IH, Qian W, et al. Cancer Cell Imaging and Photothermal Therapy in the Near-Infrared Region by Using Gold Nanorods. J Am Chem Soc. 2006;128(6):2115-2120.

2. Kalantari K, Afifi ABM, Bayat S, et al. Heterogeneou;catalysis in 4-nitrophenol degradation and antioxidant activities of silver nanoparticles embedded in Tapioca starch. Arab J Chem. 2016;12:018.

3. Singh RP, Shukla VK, Yadav RS, et al. Biological approach of zinc oxide nanoparticles formation and its characterization. Adv Mater Lett. 2011;2(4):313-317.

4. Tiwari PM, Vig K, Dennis V, et al. Functionalized Gold Nanoparticles and Their Biomedical Applications. Nanomaterials. 2011;1(1):31-63.

5. Wang Y, Xia Y. Bottom-up and top-down approaches to the synthesis of monodispersed spherical colloids of low melting-point metals. Nano Lett. 2004;4(10):2047-2050

6. Abou El-Nour KMM, Eftaiha A, Al-Warthan A, et al. Synthesis and applications of silver nanoparticles. Arab J Chem. 2010;3(3):135-140.

7. Jagpreet Singh, TS, MR. Green Synthesis of Silver Nanoparticles via Various Plant Extracts for Anti-Cancer Applications. Glob J Nano. 2017;2(3):555590.

8. Singh J, Kaur G, Kaur P, et al. A Review On Green Synthesis And Characterization Of Silver Nanoparticles And Their Applications: A Green Nanoworld. World J Pharm Pharm Sci. 2016;5(7):1.

9. Singh J, Mehta A, Rawat M, et al. Green synthesis of silver nanoparticles using sun dried tulsi leaves and its catalytic application for 4-Nitrophenol reduction. J Environ Chem Eng. 2018;6(1):1468-1474.
10. Singh J, Kukkar P, Sammi H, et al. Enhanced catalytic reduction of 4-nitrophenol and congo red dye By silver nanoparticles prepared from Azadirachta indica leaf extract under direct sunlight exposure. Part Sci Technol. 2017;0(0):1-10.

11. Singh J, Singh N, Rathi A, et al. Facile Approach to Synthesize and Characterization of Silver Nanoparticles by Using Mulberry Leaves Extract in Aqueous Medium and its Application in Antimicrobial Activity. J Nanostructures. 2017;7(2):134-140.

12. Kumar M, Mehta A, Mishra A, et al. Biosynthesis of tin oxide nanoparticles using Psidium Guajava leave extract for photocatalytic dye degradation under sunlight. Mater Lett. 2018;215:121-124.

13. Rawat M. Chemo-bio Synthesis of Silver Nanoparticles. J Nanomedicine Res. 2016;4(3):00092.

14. Savithramma N, Rao ML, Rukmini K, et al. Antimicrobial activity of Silver Nanoparticles synthesized by using Medicinal Plants. Int $J$. 2011;3(3):1394-1402.

15. Vijayakumar M, Priya K, Nancy FT, et al. Biosynthesis, characterisation and anti-bacterial effect of plant-mediated silver nanoparticles using Artemisia nilagirica. Ind Crops Prod. 2013;41(1):235-240.

16. Ramgopal M, Saisushma C, Attitalla IH, et al. A facile green synthesis of silver nanoparticles using soap nuts. Res J Microbiol. 2011;6(5):432-438.

17. Gunalan S, Sivaraj R, Rajendran V. Green synthesized ZnO nanoparticles against bacterial and fungal pathogens. Prog Nat Sci Mater Int. 2012;22(6):693-700 